\title{
Domination and Leaf Density in Graphs
}

The domination number $\gamma(G)$ of a graph $G$ is the minimum cardinality of a subset $D$ of $V(G)$ with the property that each vertex of $V(G)-D$ is adjacent to at least one vertex of $D$. For a graph $G$ with $n$ vertices we define $\epsilon(G)$ to be the number of leaves in $G$ minus the number of stems in $G$, and we define the leaf density $\zeta(G)$ to equal $\epsilon(G) / n$. We prove that for any graph $G$ with no isolated vertex, $\gamma(G) \leq n(1-\zeta(G)) / 2$ and we characterize the extremal graphs for this bound. Similar results are obtained for the total domination number. The 2-partition domination number $\gamma\left(G, \pi_{2}\right)$ of a graph $G$ and a 2-partition $\pi_{2}=\left\{V_{1}, V_{2}\right\}$ of $V(G)$ is defined by the sum $\gamma(G)+\gamma_{G}\left(V_{1}\right)+\gamma_{G}\left(V_{2}\right)$. We prove that for any graph $G$ with no isolated vertex and any 2-partition $\pi_{2}$ of $V(G)$, $\gamma\left(G, \pi_{2}\right) \leq 3 n(1-\zeta(G)) / 2$ and we characterize the extremal graphs. For graphs with leaf density $\zeta>1 / 6$, this new bound is an improvement of the bound given by B. Hartnell and P. D. Vestergaard [J. Combin. Math. Combin. Comput. 2003, Aug., 46]. 